\title{
The Chemistry of the Local Group
}

\author{
Brad K. Gibson \\ Centre for Astrophysics, University of Central Lancashire, Preston, PR1 2HE, UK \\ email: bkgibson@uclan.ac.uk
}

\begin{abstract}
Simulations of the chemical enrichment histories of ten Local Group (LG) dwarf galaxies are presented, employing empirically-derived star formation histories (SFHs), a rich network of isotopic and elemental nucleosynthetic yields, and a range of prescriptions for supernova (SN)-driven outflows. Our main conclusions are that (i) neutron-capture element patterns (particularly that of $\mathrm{Ba} / \mathrm{Y}$ ) suggest a strong contribution from low- and intermediate-mass stars (LIMS), (ii) neutron star mergers may play a relatively larger role in the nucleosynthesis of dwarfs, (iii) SN feedback alone can explain the observed gas fraction in dwarf irregulars (dIrrs), but dwarf spheroidals (dSphs) require almost all their gas to be removed via ram pressure and/or tidal stripping, (iv) the predicted heavy $\mathrm{Mg}$ isotope enhancements in the interstellar medium (ISM) of dwarfs may provide an alternate solution to claims of a varying fine structure (v) the gas lost from dwarfs have $\mathrm{O}, \mathrm{Si} / \mathrm{C}$ abundances in broad agreement with intergalactic medium abundances at redshifts $2<z<4$, and (vi) the chemical properties of dSphs are well-matched by preventing galactic winds from re-accreting, whilst those of dIrrs are better-matched by incorporating metallicity-dependent cooling and re-accretion of hot winds. Finally, doubts are cast upon a claimed association between LG dSph UMaII and HVC Complex A.
\end{abstract}

Keywords. galaxies: Local Group - galaxies: abundances - galaxies: evolution

\section{Introduction}

Due to their low mass, dwarf galaxies are unique laboratories for studying the interplay between violent feedback (eg. SN-driven outflows and starbursts), relatively quiescent inflow of both primordial and re-accreted, radiatively-cooled, metal-enhanced wind material, and the hierarchical assembly of these building blocks into more massive systems. Dwarfs may also give rise to distant high-column density gas clouds seen in absorption towards even more distant QSOs. Further, LG dwarfs are one of the few places in the Universe, outside the Milky Way, where (i) individual stellar abundances can be derived and (ii) empirical SFHs can be derived from the colour-magnitude diagrams of resolved stellar populations. Environment clearly plays an important role in shaping the SFHs and stellar populations of dwarfs, with gas-poor, predominantly old, dSphs clustered near the Milky Way, and gas-rich dIrrs, with more extended SFHs, more widely distributed.

To investigate the differences between dSphs and dIrrs, we have simulated the chemical evolution of ten LG dwarfs, exploring a wide range of prescriptions for SN feedback, cooling and re-accretion of galactic winds, and nucleosynthetic yields (including neutron capture elements). A unique aspect of our efforts is the (attempted) elimination of both star formation and infall as "free parameters". We end (Appendix A) with a cautionary note regarding the claimed association between one of the newly-discovered LG dwarfs Ursa Major II (UMaII) - and High-Velocity Cloud (HVC) Complex A.

\section{Dwarf galaxy models}

Chemical enrichment histories were simulated using an updated version of GEtool (Fenner \& Gibson 2003); enhancements included: (i) SNe-driven outflows, 
(ii) empirically-derived SFHs (Dolphin et al. 2005), (iii) semi-empirically-constrained infall rates, $\dagger$, including a provision for the incorporation of diluted, metallicity-dependent, radiatively-cooled, re-accreted wind material, and (iv) inclusion of both rapid- and slowneutron capture nucleosynthetic yields. $\ddagger$ We have relaxed the assumption that differential galactic winds act in proportion to the current star formation rate (eg. Lanfranchi et al. 2006), instead linking directly to the underlying SNe rates. We also allow for the massloading of cold, entrained, ISM gas to the SNe-driven galactic outflows, a key parameter determining the metallicity of the outflows.

\section{Results}

\subsection{Stellar Component}

Fig. 1 summarises the observed and predicted abundance ratios for 17 elements for the current sample of ten LG dwarfs. The loci of small dots in each panel correspond to simulated model stars (for our template grid of models) - for the sake of brevity, loci of both dSph and dIrr models have been overlaid. $\uparrow$ The larger symbols in each panel correspond to the extant data for each galaxy listed in the upper left panel.

Broadly speaking, the template models reproduce many of the features common to the dwarfs, including the (i) low absolute metallicities, (ii) relatively low ratios of $[\alpha, \mathrm{Sc}, \mathrm{Mn}$, $\mathrm{Cu}, \mathrm{Y} / \mathrm{Fe}]$, and (iii) trends of $[\mathrm{Ba} / \mathrm{Eu}, \mathrm{Y}]$, indicative of significant pollution from LIMS. The latter is understood in our models as a consequence of the strongly metallicitydependent s-process asymptotic giant branch (AGB) yields. At low metallicities, the ratio of the neutron flux (provided by the ${ }^{13} \mathrm{C}$ pocket) to seed nuclei is high, and the sprocess can operate efficiently to produce a bottleneck at $\mathrm{Pb}$. With increasing metallicity, there are fewer neutrons per seed nuclei and the s-process tends to produce relatively fewer heavy s-elements and more lighter s-elements. This should lead to a steep rise in $\mathrm{Ba} / \mathrm{Y}$ with increasing $\mathrm{Fe} / \mathrm{H}$ in systems with a significant AGB contribution.

General failures of the model are self-evident and reflect our poor understanding of certain stellar evolutionary nucleosynthetic pathways (note: no a posteriori scaling of the chemical evolution models or yields has been applied). These failures have been discussed by Fenner et al. (2006) and include the (i) significant overproduction of $\mathrm{Na}$ and $\mathrm{Ni}$, (ii) mild underproduction of $\mathrm{Mg}$, and (iii) inability to reach $[\mathrm{Eu} / \mathrm{Fe}] \sim+1 . \|$

\subsection{The Connection to the IGM}

Using the template models and their predicted outflow properties, we determined the redshift evolution of $[\mathrm{O}, \mathrm{Si} / \mathrm{C}]$ in the intergalactic medium (IGM), in order to compare with that observed in the IGM at redshifts $2<z<4([\mathrm{O}, \mathrm{Si} / \mathrm{C}] \approx+0.4 \rightarrow+0.7)$. The expected pollution from LG dwarfs was evaluated by summing the mass of metals ejected from our grid of dSph and dIrr models and applying a weighting factor to account for their observed frequency in the LG. The ratios thusly predicted over this redshift range match the observations regardless of stripping assumptions, and are fairly robust to model parameters and the relative number of dIrrs to dSphs.

$\dagger$ In the sense that the infall rate was "tuned" to ensure the gas density and SFHs adhered to the Kennicutt (1998) law.

$\ddagger$ The s-process yields were derived using the Torino Group's post-processing methodology, applied to the Monash Group's yields, as described by Fenner et al. (2006).

I Roughly speaking, the loci overlie one another, with the separation only becoming apparent at $[\mathrm{Fe} / \mathrm{H}]>-1$, where the dots are essentially associated only with simulated dIrr stars.

\| Alleviated by allowing a modest r-process contribution from neutron star-neutron star and neutron star-black hole binaries, as we will show in a future study; the models shown here only associate the r-process with SNe II. 


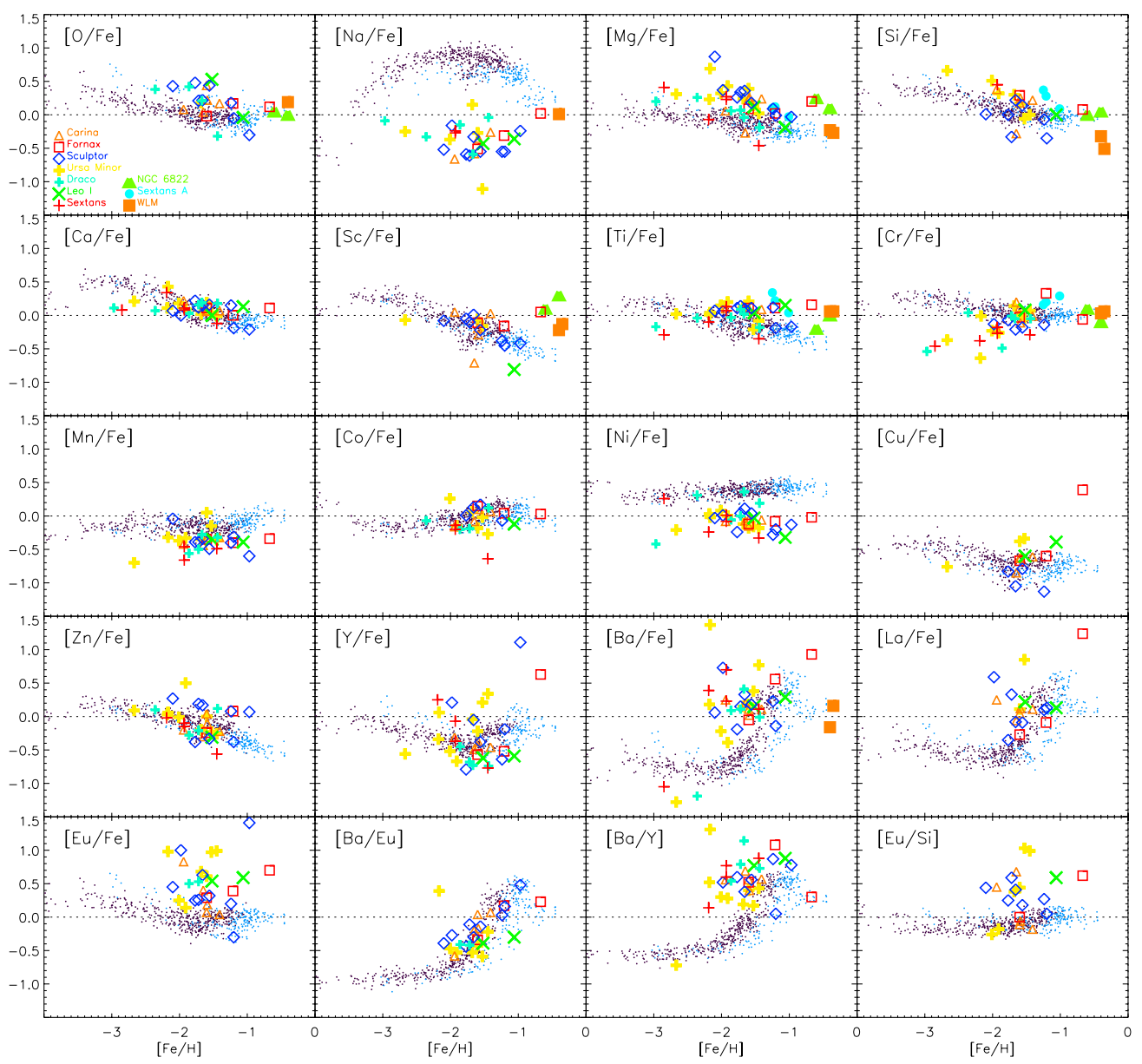

Figure 1. Predicted abundances for the ten template models, incorporating SN feedback and galactic winds (small circles), plotted against stellar observations (larger symbols). A random uncertainty of \pm 0.1 dex was imposed upon model star abundances to mimic observational errors.

\subsection{Implications for a Varying Fine Structure Constant}

It has been suggested that anomalous Mg isotopic abundances within redshift $z<2$ QSO absorbers could mimic the effect of a varying fine structure constant $\alpha$. An initial mass function (IMF) biased towards intermediate mass stars early in the evolution of a Milky Way-like system could produce the requisite factor of ten enhancement needed to reach such extreme ${ }^{25,26} \mathrm{Mg} /{ }^{24} \mathrm{Mg}$ ratios, although at the expense of introducing peculiar (and unobserved) nitrogen abundances and carbon isotope ratios (Fenner et al. 2005). Conversely, the neutral gas of our simulated dwarfs galaxies bears the imprint of LIMS pollution, provided there has been preferential loss of SNe ejecta, without the need to invoke a finely-tuned non-standard IMF. We find that the ISM of our model dSphs readily populate the enhanced ${ }^{25,26} \mathrm{Mg} /{ }^{24} \mathrm{Mg}$ regime required, but by redshift $z<2$, most are expected to have lost so much of their gas as to fall below the neutral hydrogen (HI) column density detection limit of QSO absorber studies. The better retention of gas by dIrrs could afford them greater representation in the low redshift sample. Two of our three dIrr models achieve ${ }^{25,26} \mathrm{Mg} /{ }^{24} \mathrm{Mg}$ ratios that fall within the range that could account for the varying- $\alpha$ measurement. Having said that, these two models assume that 
SNe-driven winds are permanently lost; only a modest fraction of the wind needs to be re-accreted to bring the ${ }^{25,26} \mathrm{Mg} /{ }^{24} \mathrm{Mg}$ ratio back down to near-solar.

\section{Appendix A. The Orphan Stream and HVC Complex A}

The so-called "Field of Streams" has proven to be a boon for LG dwarf studies, leading to the discovery of many new dwarfs, and the fascinating Orphan Stream (Belokurov et al. 2007). Belokurov et al, Fellhauer et al. (2007), and Jin \& Lynden-Bell (2007) each note the spatial and kinematical coincidence of HVC Complex A with the predicted orbit of one of these new dwarfs, UMaII. with Fellhauer et al. making the reasonable suggestion (via the use of N-body simulations) that the progenitor of UMaII was likely to have had a dynamical mass of $\sim 3 \times 10^{5} \mathrm{M}_{\odot}$ (and certainly $<10^{6} \mathrm{M}_{\odot}$ ) and now lies at a heliocentric distance of $\sim 30 \mathrm{kpc}$ (with an absolute magnitude of $\sim-7.5$ ). While we find it likewise tempting to associate UMaII and the Orphan Stream with Complex A, the latter association appears difficult to reconcile with the following points:

- Complex A lies 4-10 kpc from the Sun, and not $30 \mathrm{kpc}$

- assuming UMaII adheres to the luminosity-metallicity relation, much like the other LG dSphs, one would expect a metallicity $\ll 1 \%$ solar, for its luminosity, while Complex A almost certainly possesses a metallicity $>10-20 \times$ that value

- the $\alpha / \mathrm{Fe}$ ratio within Complex $\mathrm{A}$ is likely enhanced, as in Complex $\mathrm{C}$, and consistent with a SNeII signature, while present-day LG dSphs typically present sub-solar $\alpha / \mathrm{Fe}$

- perhaps most difficult to reconcile is the fact that at a putative distance of $30 \mathrm{kpc}$, the HI mass of Complex A would be $\sim 10^{7} \mathrm{M}_{\odot}$ (even neglecting the known and substantial ionised gas components associated with Complex A), $\sim 30 \times$ the dynamical mass of the putative UMaII progenitor, alone. Even at the lowest allowable distance $(4 \mathrm{kpc})$, the HI mass of Complex A already equates to the putative UMaII progenitor's dynamical mass, leaving no room for a dark matter or stellar component.

- a further $\sim 10^{6} \mathrm{M}_{\odot}$ of stars would also be required to enrich the $10^{7} \mathrm{M}_{\odot}$ of gas to $10 \%$ solar metallicity, only exacerbating this mass "problem"

In consort, while it is tempting to link Complex A with UMaII and the Orphan Stream, the problem appears more complicated than one of simply recovering spatial and kinematical coincidence!

\section{Acknowledgements}

This work could not have been undertaken without the remarkable skills of Yeshe Fenner; her contributions are gratefully acknowledged, as is the ongoing guidance of Leticia Carigi, Andrea Marcolini, Stéphanie Courty, and Patricia Sánchez-Blázquez.

\section{References}

Belokurov, V., Evans, N.W., Irwin, M.J., et al. 2007, ApJ in press, astro-ph/0605705

Dolphin, A.E., Weisz, D.R., Skillman, E.D. \& Holtzmann, J.A. 2005, preprint astro-ph/0506430

Fellhauer, M., Evans, N.W., Belokurov, V., et al. 2007, MNRAS 375, 1171

Fenner, Y. \& Gibson, B.K. 2003, PASA 20, 189

Fenner, Y., Murphy, M.T. \& Gibson, B.K. 2005, MNRAS 358, 1337

Fenner, Y., Gibson, B.K., Gallino, R. \& Lugaro, M. 2006, ApJ 646, 184

Kennicutt, R.C. 1998, ApJ 498, 541

Lanfranchi, G.A., Matteucci, F. \& Cescutti, G. 2006, MNRAS 365, 477

Shin, J. \& Lynden-Bell, D. 2007, preprint astro-ph/0703397 